Research Article

\title{
In vitro evaluation of virulence factors associated with clinical isolates of Candida species from patient in tertiary care hospital in Central India
}

\author{
Gupta $\mathbf{J}^{\mathbf{1}}$, Shah $\mathbf{H}^{\mathbf{2}}$ \\ ${ }^{1}$ Dr Jyoti Gupta, Assistant Professor, Department of Microbiology, Chirayu Medical College, Bhopal, MP, ${ }^{2}$ Dr. Harshada \\ Shah, Prof and Head, Department of Microbiology, R. D. Gardi Medical College, Surasa, Ujjain, M.P, India.
}

Address for Correspondence: Dr Jyoti Gupta, Assistant Professor, Department of Microbiology, CMCH, Bhopal, MP, Email: drjyoti93@gmail.com

\begin{abstract}
Background: Adherence to host tissues, morphological changes and secretion of extracellular hydrolytic enzymes are important virulence attributes of Candida species. These enzymes play pivotal roles in pathogenicity of Candida infection. Aim: The present study was conducted with an aim to determine in vitro phospholipase, haemolysin, Coagulase activities and biofilm production of Candida species isolated from various Clinical samples. Material and Method: A total of 156 Candida species isolated from various clinical specimens were identified up to species level by standard mycological techniques and was tested for extracellular hydrolytic enzyme activity and Biofilm formation. Results: 156 Candida species were isolated from 915 clinical specimens. Biofilm production was shown by 77 candidal strains (49.35\%), phospholipase activity was observed by 74 candidal strains $(47.43 \%)$. haemolysin activity was observed by 66 candidal strains $(42.30 \%)$ and Coagulase production in 38 of Candida (24.35\%) isolates. Conclusion: Both $C$. albicans and Non-albicans Candida (NAC) species produce extracellular hydrolytic enzymes. Since these enzymes are important to understand the co-relation between the species and infection, their detection is extremely important in the management of infections caused by various Candida species.
\end{abstract}

Keywords: Extracellular hydrolytic enzymes, phospholipase, haemolysin, Coagulase non-albicans Candida species.

\section{Introduction}

The incidence of invasive fungal infection has increased steadily over the past two decades. With recent advances in medical and surgical intervention and increasing population of immunocompromised patients, the diversity and list of fungal pathogens continue to grow [1]. The virulence attributes of Candida includes hyphal switching, germination, surface recognition and production of extracellular hydrolytic enzyme [2]. Among these, extracellular hydrolytic enzymes play an important role in adherence and infection of Candida [3]. Recently, microbial biofilms have gained prominence because of the increase in infections related to indwelling medical devices. The advantages of forming a biofilm for the organism include protection from the environment, nutrient availability, metabolic cooperation, and acquisition of new genetic traits [4].

Manuscript received: $28^{\text {th }}$ April 2017

Reviewed: $8^{\text {th }}$ May 2017

Author Corrected: $17^{\text {th }}$ May 2017

Accepted for Publication: 24 $4^{\text {th }}$ May 2017
Research on Candida pathogenicity has been focused on hydrolytic enzymes, such as proteinases, phospholipases and lipases. Little information concerning Candida coagulase and hemolytic activities was found in a recent literature search.

Hemolytic and coagulase activities in Candida spp. are virulence factors less well-studied and their importance to pathogenicity require new and more rigorous studies [5].

The present study was conducted with an aim to determine in vitro phospholipase, haemolysin, Coagulase activities and biofilm production of Candida species isolated from various Clinical samples.

Aim: To determine the virulence factors of Candida species isolated from various clinically suspected samples of Candidiasis. 


\section{Materials and Methods}

Study design- Hospital based cross sectional study.

Study setting- Department of Microbiology, Ruxmaniben Deepchand Gardi Medical College (RDGMC) and Chandrikaben Rashmikant Gardi Hospital (CRGH) and Research Centre, Surasa, Ujjain.

Study subjects- Clinically suspected candidiasis among patients admitted in C.R.Gardi Hospital.

Study samples- Clinical samples from suspected patients of candidiasis including-urine, blood, vaginal swab, oral swab, sputum, stool, lip scraping, pus, CSF, central line tip, eye swab and umbilical swab.

Study period- One and a half year from January 2013 to June 2014.

915 Clinical samples from suspected patients of candidiasis received in Laboratory. 156 Candida species were isolated from 915 clinical specimens.

The study was approved by the Institutional research and ethics committee. Speciation of Candida isolates were done by evaluating the germ tube formation, assimilation, and sugar fermentation test. Culture on cornmeal agar was for demonstration of chlamydospores while culture on candid chromagar was for the identification of the species. 156 isolates were screened for extracellular enzymes production and Biofilm formation.

\section{Determination of virulence factor}

1) Phospholipase enzyme production

2) Haemolytic enzyme production

3) Coagulase production

4) Production of biofilm

1) Phospholipase enzyme production: The phospholipase activity of Candida isolates was detected by the method of Samaranayakeet al. [6] (color plate-1)

Preparation of media- The egg-yolk medium consisted of $13.0 \mathrm{~g}$ Sabouraud's dextrose agar (SDA), $11.7 \mathrm{~g}$ sodium chloride, $0.11 \mathrm{~g}$ Calcium chloride and $10 \%$ sterile egg yolk in $184 \mathrm{ml}$ distilled water. First, the components without the egg yolk were mixed and sterilized (autoclave). After autoclaving the egg yolk was centrifuged at $500 \mathrm{rpm}$ for 10 minute at room temperature and $20 \mathrm{ml}$ of the supernatant was added to the sterilized medium.
Yeast suspension preparation $-10^{8}$ yeast cells $/ \mathrm{ml}$ was prepared in sterile saline

Procedure- Extracellular phospholipase activity was detected by inoculating $5 \mu \mathrm{l}$ of yeast suspension onto the surface of the egg-yolk medium and left to dry at room temperature. Candida albicans 90028 was used as Positive control. Culture plate was incubated at $37^{\circ} \mathrm{C}$ for 2 days.

Calculation of the zone of phospholipase activity $(\mathrm{Pz})$ was performed as follows:

$$
\mathrm{Pz}=\frac{\text { Colony Diameter }}{\text { Colony Diameter }+ \text { Zone of precipitation }}
$$

- Pz ratio of one- no activity: negative (-)

- Pz ratio of less than one - indicated the phospholipase activity.
a) $\quad 0.99-0.9$ as weak $(+)$
b) $0.89-0.8$ as mild $(++)$
c) $0.79-0.7$ as relatively strong $(+++)$
d) $<0.69(++++)$ as very strong positive

The lower the $\mathrm{Pz}$ value, higher the phospholipase activity $[7,8]$.

Haemolytic enzyme production: Haemolysin activity was assessed by the method of Manns et al. [9] on blood agar plate. (color plate-2)

Media preparation- SDA supplemented 7\% sheep blood and $3 \%$ glucose with a final $\mathrm{pH}$ adjusted to $5.6 \pm$ 0.2 was used.

Procedure- $10 \mu \mathrm{l}$ of previously prepared yeast suspension was inoculated onto plates and incubated at $37{ }^{\circ} \mathrm{C}$ in $5 \% \mathrm{CO}_{2}$ for $48 \mathrm{~h}$.

Interpretation- Beta $(\beta)$-haemolysis was indicated by a clear, colourless zone surrounding the colony of the organism and alpha $(\alpha)$-haemolysis showed a greenish zone. Gamma $(\gamma)$ haemolysis was indicated by an absence of any apparent haemolysis or discolouration. To represent the extent of haemolysin activity by different Candida isolates [Haemolysin activity $(\mathrm{Hz})$ was determined by calculating the ratio of the diameter of the colony to that of the translucent zone of haemolysis (in $\mathrm{mm}$ ). The assay was conducted on three 
separate occasions for each isolate. C. albicans ATCC 90028 was used as positive control. One strain each of Streptococcus pyogenes (Lancefield group A) and Streptococcus sanguis, which induce beta and alpha haemolysis, were used as positive controls. $[5,10,11]$.

3) Coagulase production of Candida spp: was done by EDTA- Sheep plasma by a tube test method $0.1 \mathrm{ml}$ of an overnight culture of each isolate in Sabouraud's dextrose broth was inoculated into a tube containing $500 \mu 1$ of EDTA- Sheep plasma. The tubes were incubated for $4 \mathrm{~h}$ at $35^{\circ} \mathrm{C}$. The presence of a clot that could not be resuspended by gentle shaking indicated positive coagulase test. If no clot formed, the tube was reincubated \& e-examined after 24 h. Staphylococcus aureus ATCC 25923 and S. epidermidis ATCC 14990 were used as positive and negative controls. [5,12] (color plate-3)

4) Detection of biofilm production - Biofilm formation by Candida isolates was assessed by the tube method as described by et al. A loopful of yeast from overnight SDA culture plates was inoculated in $10 \mathrm{ml}$ Sabouraud's dextrose broth in polypropylene falcon tube and incubated for $48 \mathrm{~h}$ at $35^{\circ} \mathrm{C}$. After $48 \mathrm{~h}$, broth was aspirated gently and washed with distilled water twice and then stained with $1 \%$ safranin for 10 min after which they were examined on walls for the presence of an adherent layer. Biofilm producer Staphylococcus epidermidis ATCC 35984 was used as a positive control. Each isolate was tested twice and read independently by two different observers. [5,13] (color plate-4)

Interpretation: Biofilm formation was considered positive when a film lined the wall and bottom of the tube. Ring formation at the liquid interface was not considered as an indication of biofilm production $[8$, $10]$.

Biofilm production was scored as

- $\quad 0 \ldots . . .$. non adherent(NA),

- $\quad+\ldots \ldots . .$. weak adherent (WA)

- $\quad++\ldots \ldots . . .$. Moderate adherent (MA)

- $\quad+++\ldots \ldots . . .$. strong adherent (SA)

\section{Result}

Table-1: Phospholipase activities of different Candida species $(n=156)$.

\begin{tabular}{|c|c|c|}
\hline Species & No of isolates & Phospholipase enzyme Production \\
\hline C.tropicalis & 73 & $43(58.90 \%)$ \\
\hline C.albicans & 58 & $21(36.20 \%)$ \\
\hline C.parapsilosis & 15 & $7(46.66 \%)$ \\
\hline C.kefyr & 3 & 0 \\
\hline C.guillermondii & 3 & $1(33.33 \%)$ \\
\hline C.krusei & 2 & $2(100 \%)$ \\
\hline C.dublienensis & 2 & 0 \\
\hline Total & 156 & $74(47.43 \%)$ \\
\hline
\end{tabular}

In present study C.tropicalis shows the maximum Phospholipase activity. Phospholipase activity was seen in $54.08 \%$ NAC species and $36.20 \%$ C.albicans species.

Table-2: Extracellular hydrolytic activity of different Candida species.

\begin{tabular}{|c|c|c|c|}
\hline Species & No of isolates & Haemolysin Production & Coagulase Production (Sheep Blood) \\
\hline C.tropicalis & 73 & $37(50.68 \%)$ & $21(28.76 \%)$ \\
\hline C.albicans & 58 & $27(46.55 \%)$ & $15(25.86 . \%)$ \\
\hline C.parapsilosis & 15 & $1(6.66 \%)$ & 0 \\
\hline C. kefyr & 3 & 0 & 0 \\
\hline C.guillermondii & 3 & 0 & 0 \\
\hline C.krusei & 2 & 0 & 0 \\
\hline C.dublienensis & 2 & $1(50 \%)$ & $\mathbf{3 8}(\mathbf{2 4 . 3 5 \% )}$ \\
\hline Total & $\mathbf{1 5 6}$ & $\mathbf{6 6}(\mathbf{4 2 . 3 0} \%)$ & 0 \\
\hline
\end{tabular}


Research Article

I) Haemolysin activity (Hz) - Among all the isolates, 66 (42.30\%) expressed hemolytic activity. Beta hemolysis was seen. The hemolytic index values ranged from $0.32-0.86$.

Haemolysin production was more in NAC (39.79\%). Haemolysin production was not noted in C.kefyr, C.krusei \& C.guillermondii.

II) Coagulase production- Maximum Coagulase production was seen in C.tropicalis (28.76\%) isolates in sheep plasma

Table-3: Slime production by Candida species ( $\mathrm{n}=156)$

\begin{tabular}{|c|c|c|c|c|c|}
\hline Species & $\begin{array}{c}\text { No of } \\
\text { Isolates }\end{array}$ & No of positive isolates & $\begin{array}{c}\text { Weakly } \\
\text { adherent } \\
(\mathbf{1 +})\end{array}$ & $\begin{array}{c}\text { Moderately } \\
\text { adherent } \\
\mathbf{2 +}\end{array}$ & $\begin{array}{c}\text { Strongly } \\
\text { adherent } \\
(\mathbf{3}+)\end{array}$ \\
\hline C.tropicalis & 73 & $42(57.53 \%)$ & 13 & 12 & 17 \\
\hline C.albicans & 58 & $24(41.37 \%)$ & 14 & 2 & 8 \\
\hline C.parapsilosis & 15 & $8(53.33 \%)$ & 2 & 3 & 3 \\
\hline C.krusei & 2 & $1(50 \%)$ & 0 & 1 & 0 \\
\hline C.guillermondii & 3 & $1(33.33 \%)$ & 0 & 1 & 0 \\
\hline C.kefyr & 3 & 0 & 0 & 0 & 0 \\
\hline C.dublienensis & 2 & $1(50 \%)$ & 0 & 1 & 0 \\
\hline Total & $\mathbf{1 5 6}$ & $\mathbf{7 7 ( 4 9 . 3 5 \% )}$ & $\mathbf{2 9}$ & $\mathbf{2 0}$ & $\mathbf{2 8}$ \\
\hline
\end{tabular}

C.tropicalis (57.53\%) was the most common biofilm producer

\section{Discussion}

We have done study on selective virulence factors such as phospholipase, hemolysin, coagulase enzyme production and biofilm.

Out of 156 Candida species studied, phospholipase activity was seen in 74 (47.43\%) isolates. Previous studies have reported phospholipase activity in 30 to $100 \%$ of Candidal isolates. Similar observations were found in other Indian studies [14]. Vinita et al study indicates that even though all the isolated strains were pathogenic, not all strains of Candida produced phospholipase as virulent factors. The virulence of Candida species is attributed not to a single factor but to a combination of several factors, like proteinase, phospholipase, biofilm production etc. Phospholipase positivity among non-albicans Candida strains was reported by Vinita et al [14] and Sachin et al [11]. In present study among the NAC species $C$. tropicalis showed maximum phospholipase production $(58.90 \%)$, so it means it is more invasive among various isolates. Similar observations were found in other Indian studies [15]. In our study Phospholipase was the major virulent factor expressed by $C$. tropicalis isolates. Researchers have reported contradictory findings regarding phospholipase activity in $C$. tropicalis. Investigators like Thangam et al [16] reported high phospholipase activity in $C$. tropicalis isolates among NAC spp. while others like Samaranayake et al [17] reported no activity. These inconsistencies in observations may be due to biological differences among the isolates tested [15].

Most of the studies on exoenzymes are focused on phospholipases and secreted aspartyl proteinases (Sap). Coagulase production and haemolytic activity of are the least studied [12].

In present study haemolysin activity was detected in $42.30 \%$ isolates of Candida species. Study by Sachin et al [1] in 2012 noted $(51.8 \%$ ) haemolytic activity and $48 \%$ in C. tropicalis. In our study all isolates showed $\beta$ type of haemolysis and $C$. tropicalis showed maximum haemolytic activity (50.68\%). Haemolysin is another putative virulence factor thought to contribute to candidal pathogenesis. In particular, the secretion of haemolysin, followed by iron acquisition, facilitates hyphal invasion in disseminated candidiasis [5]. 
Research Article

In the present study $24.35 \%$ isolates were coagulase test positive when tested with sheep plasma. None of the these Candida isolates reacted positively in human plasma. In the study by Sachin et al [11] in 2013 reported 30.1\% isolates were reported coagulase positive. Coagulase binds plasma fibrinogen and activates a cascade of reactions that induce clotting of plasma. Rodrigues et al. [18] reported high coagulase activity in C.tropicalis (82.6\%). Variations in coagulase production by Candida spp may be related to their pathogenicity. It is concluded that the laboratory detection of coagulase activity in clinical isolates of Candida spp may help the diagnosis of Candida-related infections.

Among the three virulence factors, Phospholipase (47.43\%) was the most common virulent factor followed by Haemolysin production (42.30\%) and Coagulase production (24.35\%) in the present study.

NAC species 53/98 (54.08\%) produced biofilm in comparison to C. albicans $24 / 58(41.37 \%)$ showing relatively lower slime production. Also strong biofilm production was seen in cases of $C$. tropicalis compared to weak biofilm production that was seen in C. kefyr, C. krusei, and C.guillermondii.

Similar observation was seen in study by Yigit et al.[5] Studies, investigating biofilm production among Candida species detected higher levels of positivity among non-albicans Candida strains (51-60\%) than that of C.albicans strains (8-12\%) [8]. Biofilms are surface associated microbial communities which are firmly fixed within an extracellular matrix [19]. It limits the penetration of an antifungal agent through the matrix and protects the yeast cells from host immune response.

Virulence of Candida depends on the type of species and the clinical sample from which it has been isolated. Hence, significance of virulence determination in rational antifungal therapy cannot be ignored and should be adopted as a routine procedure in the laboratory.

\section{Conclusion}

In conclusion, it was seen that NAC spp. had greater biofilm-forming ability when compared to C. albicans species especially $C$. tropicalis showed the highest ability to produce phospholipase, haemolysin, Coagulase and biofilms. Species of Candida other than C. albicans which were previously considered as less or non virulent are now implicated in human disease. In our study extracellular hydrolytic enzyme activities was shown by $C$. albicans and NAC species. Detection of virulence factors like phospholipase, haemolysin, Coagulase and Biofilm formation will help to provide sufficient data in the establishment of a relationship between the infection and Candida species isolated.

This study will provide data about distribution of various Candida species and their Virulence markers among patients visiting our tertiary care hospital and our geographical area specially in and around Ujjain district (M.P.).

\section{Detection of Virulence factor}

Color plate-1

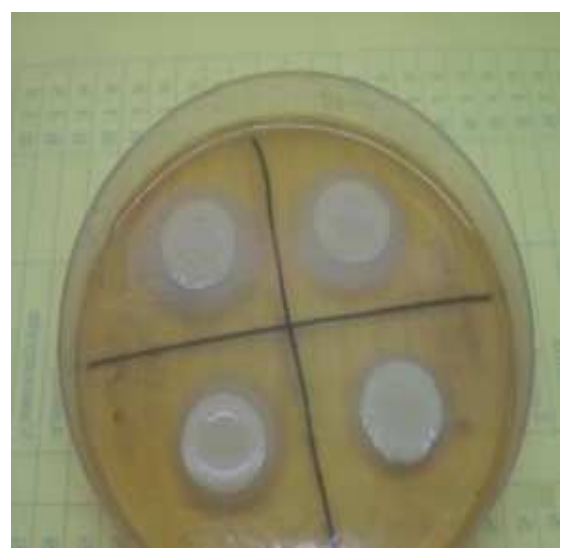

Phospholipase enzyme Production
Color plate -2

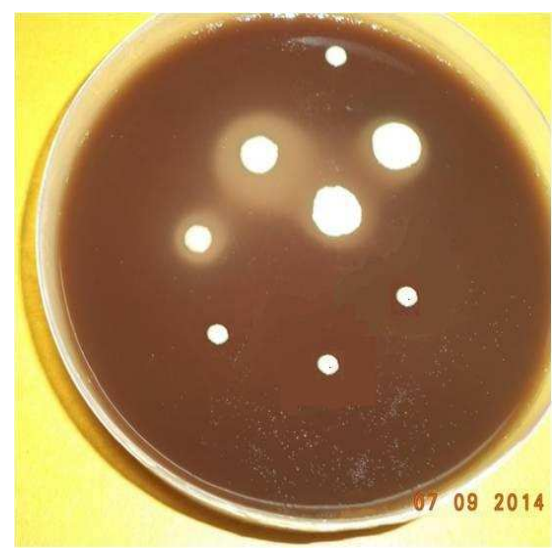

Haemolysin enzyme production 
Color plate -3

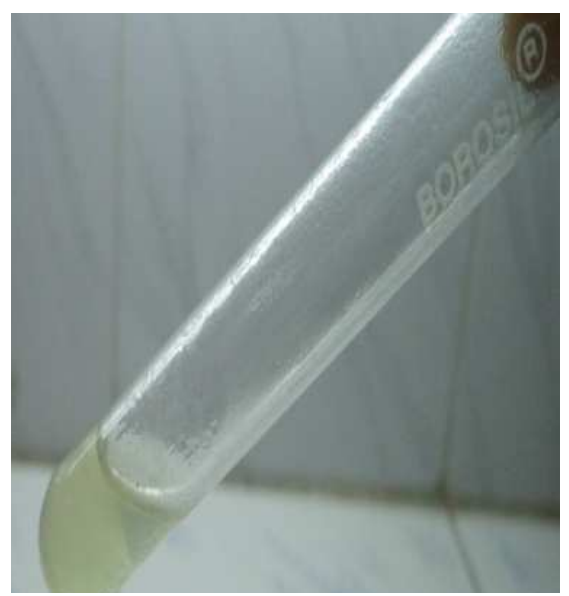

Positive
Coagulase production

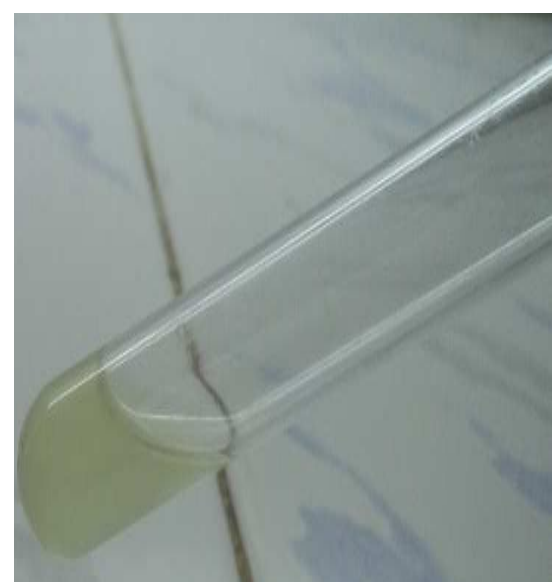

Negative

\section{Color plate-4 Biofilm formation}

a) Non-adherent b)weakly-adherent c)Moderately-adherent d)Strongly adherent

a

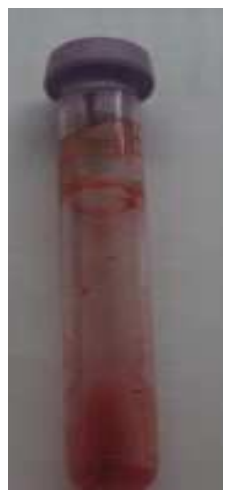

b

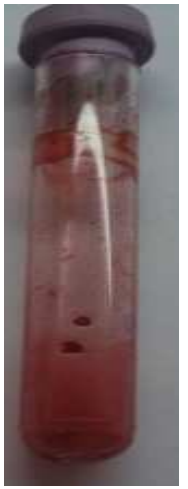

c

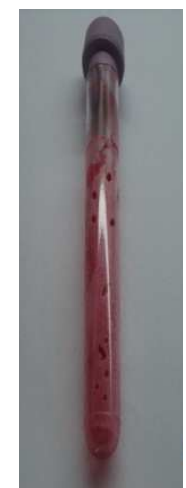

d

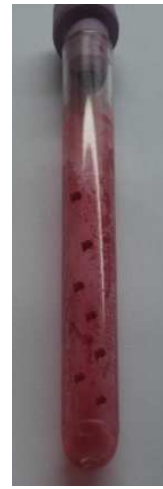

Funding: Nil, Conflict of interest: None initiated,

Permission from IRB: Yes

\section{References}

1. Sachin C.D, Ruchi K, Santosh S. In vitro evaluation of proteinase, phospholipase and haemolysin activities of Candida species isolated from clinical specimens. Int J Med Biomed Res 2012;1(2):153-7.

2. Ying S, Chunyang L. Correlation between phospholipase of Candida albicans and resistance to fluconazole. Mycoses Jan2012; 55(1): 50-5 doi:10.1111/j.1439-0507.2011.02024.x

3. Deorukhkar SC, Saini S. Non albicans Candida species: Its isolation pattern, species distribution, virulence factors and antifungal susceptibility profile. Int J Med Sci Public Health 2013; 2(3):533-8. doi: 10.5455/ijmsph.2013.080320131
4. Gwan V, Butola R, Madan M. Comparison of Biofilm formation in clinical isolates of Candida species in a tertiary care center, North India. Indian J Pathol Microbiol 2015; 58 (4): 475-8.

5. Yigit, et al. Investigating Biofilm Production, Coagulase and Hemolytic Activity in Candida Species Isolated From Denture Stomatitis Patients Eurasian J Med. 2011; 43(1): 27-32.

6. Samaranayake LP, Raeside JM, MacFarlane TW. Factors affecting the phospholipase activity of Candida species in vitro. Sabouraudia. 1984; 22 (3): 201-7. 
7. Deorukhkar S, Saini S. Evaluation of phospholipase activity in biofilm forming Candida species isolated from intensive care unit patients. British Microbiology Research Journal. 2013;3(3):440-447.

8. Kumari V, Banerjee T, Kumar P, Pandey S, Tilak R. Emergence of non-albicans Candida among candidal vulvovaginitis cases and study of their potential virulence factors, from a tertiary care center, North India. Indian J Pathol Microbiol 2013; 56(2):144-7.

9. Manns MJ, Mosser MD, Buckley RH. Production of a hemolytic factor by Candida albicans. Infect Immun. 1994;62(11):5154-6.

10. Ebhodaghe B, Ajibade K. Identification of Virulence Factors Produced by Candida Isolates from HIV Seropositive and HIV Seronegative Pregnant Women. IJTDH.2016; 20(1): 1-9.

11. Deorukhkar S, Saini S. Non albicans Candida species: its isolation pattern, species distribution, virulence factors and antifungal susceptibility profile. Intern $\mathbf{J}$ of Med Science and Public Health. 2013; 3 (2):533-38.

12. Yigit, N, Aktas A, Ayyildiz A. Detection of Coagulase Activity in Pathogenic Candida Species. J of Int Medical Research 2008;36(6): 1378-1382

13. Mohandas V. Distribution of Candida species in different clinical samples and their virulence: Biofilm formation, proteinase and phospholipase production: A study on hospitalized patients in Southern India. J Glob Infect Dis. 2011; 3(1):4-8

14. Mohan das V, Ballal M. Proteinase and phospholipase activity as virulence factors in Candida species isolated from blood. Rev Iberoam Micol. 2008 Dec 31; 25(4):208-10.

15. Deorukhkar SC, Saini S, Mathew S. Virulence Factors Contributing to Pathogenicity of Candida tropicalis and its antifungal Susceptibility Profile. Int J of Microbiol, vol. 2014, Article ID 456878,6pages, doi: $10.1155 / 2014 / 456878$

16. Thangam M, Smitha S. Deivanayagam, C. N. Phospholipase activity of Candida isolates from patients with chronic lung disease. Lung India. 1989; 7 (3): 125-6.

17. Samaranayake LP, Raeside JM, MacFarlane TW. Factors affecting the phospholipase activity of Candida species in vitro. Sabouraudia. 1984;22(3):201-7.

18. Rodrigues AG, Pina-Vaz C, Costa-De-Oliveira S, Tavares C. Expression of plasma coagulase among pathogenic Candida species.J of Clinical Microbiology. 2003; 41(12):5792-3.

19. Deorukhkar SC, Saini S. Non albicans Candida species: A review of epidemiology, pathogenicity and antifungal resistance Pravara Med Rev 2015; 7(3):7-15.

\section{How to cite this article?}

Gupta J, Shah H. In vitro evaluation of virulence factors associated with clinical isolates of Candida species from patient in tertiary care hospital in Central India. Trop J Path Micro 2017;3(2):188-194.doi: 10.17511/jopm.2017.i2.20. 